\title{
How Hormones Influence Composition and Physiological Function of the Brain-Blood Barrier
}

\author{
R. HAMPL ${ }^{1}$, M. BIČÍKOVÁ ${ }^{1}$, L. SOSVOROVÁ ${ }^{1}$ \\ ${ }^{1}$ Institute of Endocrinology, Prague, Czech Republic
}

Received June 18, 2015

Accepted July 2, 2015

\begin{abstract}
Summary
Hormones exert many actions in the brain. Their access and effects in the brain are regulated by the blood-brain barrier (BBB). Hormones as other substances may enter the brain and vice versa either by paracellular way requiring breaching tight junctions stitching the endothelial cells composing the BBB, or by passage through the cells (transcellular way). Hormones influence both ways through their receptors, both membrane and intracellular, present on/in the BBB. In the review the main examples are outlined how hormones influence the expression and function of proteins forming the tight junctions, as well as how they regulate expression and function of major protein transporters mediating transport of various substances including hormone themselves.
\end{abstract}

\section{Key words}

Blood-brain barrier • Tight junction proteins • Paracellular way • Protein transporters $\bullet$ Hormones

\section{Corresponding author}

R. Hampl, Institute of Endocrinology, Národní 8, 116 94, Prague 1, Czech Republic. E-mail: rhampl@endo.cz

\section{Introduction}

The brain is a privileged organ coordinating and controlling main physiological functions including central nervous activity. As such it must be supplied with a sufficient energy and protected from undesired endogenous or environmental noxious substances. Indeed, the brain, though amounting only about $2 \%$ of the total body weight, it consumes as much as $18 \%$ of the whole energy. One of the tools ensuring these functions is the blood-brain barrier (BBB). It is an interface on the outer site of brain vessels, separating the brain extracellular fluid and brain parenchymal cells from the blood stream. Anatomically it is formed by capillary endothelial cells, to which, from the brain side adhere glial cells - astrocytes and pericytes (Tajes et al. 2014).

Endothelial cells are rich of a plenty of transmembrane and other proteins - ion channels, receptors, transportes and enzymes, some of which are specific for the cells forming the BBB. We distinguish the luminal (blood-facing) and abluminal (brain-facing) membranes, the composition and function of which may differ. The endothelial cells forming the BBB differ from other endothelial cells throughout the body not only by presence of more or less specific transporters and receptors, but also by tight junctions (TJ) between the cells, not allowing passage of molecules across the membrane. These junctions thus effectively prevent diffusion through the intercellular pores. They are composed of proteins, usually dimers, anchored in endothelial cell membranes, often to other proteins. Examples of such proteins are occludin, claudins and many other proteins, commonly termed junctional adhesion molecules (JAM). The characteristic feature of the tight junctions is an extremely high electric resistance (Abbott et al. 2010).

In the following paragaphs we will briefly summarize the main ways how various substances may get across the $\mathrm{BBB}$, with a particular attention to hormones, with respect to their pluripotent actions in the brain. Finally we will show, how various hormones affect the function and composition of the BBB. 


\section{Transport of substance across the BBB}

Generally, compounds (and in some instance even also cells) may get across the BBB by two ways: 1. By breaching tight junctions by which the cells are "stitched" (paracellular way). 2. By passing through the epithelial cells (transcellular pathway). Breaching, or more generally, impairment or even disruption of the $\mathrm{BBB}$, is a typical feature of the most severe neurodegenerative diseases as Alzheimer's or other dementias, multiple sclerosis and parkinsonism. Mechanisms of transcellular transport are more sophisticated events, with a number of various participating actors, as shown in the next text.

The main transcellular pathways include: 1. Passive diffusion in the direction of the concentration gradient. It includes carbon dioxide, water, with some exceptions due to existence of ion channels, the major electrolytes and a broad group of small lipid-soluble molecules including low-molecular hormones as unconjugated steroids. Generally, hydrophilic substances and compounds of high molecular weight do not possess the physico-chemical properties required for passive diffusion. 2. Carrier-mediated transport using transporters as transmembrane proteins on the luminal or abluminal membrane, or eventually, on both. A facilitated transport not consuming energy as well as active transport may occur here. 3. Transport based on ligand-membrane receptor interaction. A specific case of the latter represents transport of the ligands mediated by the receptor for plasma transport protein to which the ligand is bound (Hampl et al. 2014).

The transport of major nutrients - glucose, lipids and amino acids, depends on their molecular weight and physico-chemical properties. Many of them have their own more or less specific transporters. Glucose supply to the brain is of crucial importance, and the $\mathrm{BBB}$ is abundant in membrane glucose transporters, the number of which, in contrast to other cells is mostly independent on insulin (Barros et al. 2007).

Most (but not all) lipoproteins are transported to and out of the BBB by using plasma lipoprotein carriers and their membrane receptors. In contrast to transport from circulation to other tissues, only high-density lipoproteins (HDL) appear to traverse the BBB (Wang and Ecke 2014). Among the lipids, brain cholesterol, amounting as much as almost $20 \%$ of the entire body's cholesterol is of particular importance because it is an essential constituent of myelin forming neuronal sheaths.
The blood-borne cholesterol, however, can only with difficulty traverse the $\mathrm{BBB}$; in fact, only its minor portion uses plasma lipoproteins, as it is common for cholesterol transport from blood to other tissues. Therefore most of the brain cholesterol must be formed there de novo (Jeitner et al. 2011). Cholesterol in the brain undergoes intensive metabolism, including formation of neurosteroids. Regarding their transport across the BBB, of importance are cholesterol hydroxyderivatives (oxysterols), which can freely cross the BBB. While the major metabolite for efflux is 24S-hydroxycholesterol, in the opposite direction it is 27-hydroxycholesterol. This remarkable mechanism helps to maintain the brain cholesterol homeostasis (Björkhem 2006).

Different transporters were described for neutral as well as charged amino acids (Smith 2000, Hawkins et al. 2006). A large transport protein superfamily transporting also anionic amino acids and thyroid hormones are known as organic anion transporting polypeptides, abbreviated OATPs (Wirth et al. 2014). These transmembrane proteins mediate the translocation of a broad spectrum of amphipathic substrates as anionic polypeptides and also many drugs, toxins and xenobiotics and, last but not least, also steroid conjugates. They are expressed in many tissues, not only in endothelial cells forming the BBB (Hagenbuch and Meier 2003).

Generally, peptides and proteins from blood stream, including a large number of peptide hormones, can overcome the BBB only with difficulty by simple diffusion. In most instances their transport is ensured by selective transporters, which, in concert with enzymes on both sides of the endothelial membrane, can modify the permeability for these substances (Banks 2008a). An important superfamily of transmembrane transporters, with a very broad substrate specificity, are proteins of the so called ATP-binding cassette (ABC). They occur not only on the $\mathrm{BBB}$, but on the most of endothelial cells. Permeability glycoprotein or P-glycoprotein (pgP) is of particular importance there. Its role consists in prevention of flux of some lipophilic drugs into the brain and, at the same time, it facilitates the removal of many substances from the brain, both in an ATP-dependent manner. Proteins of the ATP-binding cassette were tested with a view to support the clearance of neurotoxic peptides accumulating in the brain such as amyloid- $\beta$ (A $\beta$ ) peptides in Alzheimer's disease (Löscher and Potschka 2005, Shen and Zhang 2010).

As mentioned already, glucose transport to the brain does not depend on insulin, but insulin acts there as 
an important regulator of central glucose metabolism. Via the insulin receptor substrate present in the brain cells, it initiates signaling pathways leading to the regulation of not only peripheral metabolism, but also feeding behavior and memory and thus contributes to maintenance of many neural functions, such as neuronal growth, differentiation, neuromodulation and neuroprotection (Ramalingam and Kim 2014). Circulating insulin crosses the BBB using its own saturable transporter. The physico-chemical properties of the insulin transporter may be affected by pathological factors such as hyperglycemia and diabetes. Insulinoresistance within central nervous systems is typical for Alzheimer's dementia (Banks et al. 2012).

Besides insulin, there is an array of peptide hormones controlling energy homeostasis such as the white adipose tissue-produced leptin, adipokines and gastric ghrelin. They control appetite and thermogenesis in the brain, resulting in a decrease in obesity. Leptin mediates a negative feedback loop between the adipose tissue and brain (Kalra and Kalra 2010). Leptin and peptides of this group use their own receptor-transporters to cross the BBB (Pan and Kastin 2007). A failure of the leptin transporter in the $\mathrm{BBB}$ may result in leptin resistance in obese subjects (Banks 2008b).

When referring to peptides passing across the $\mathrm{BBB}$, we must not leave out a multifarious array of cytokines. It should be pointed out that they not only pass across the $\mathrm{BBB}$ from the circulation, but that they are synthesized in the brain as well, and even that they are expressed in the BBB endothelian cells. Through their receptors on both sides of the $\mathrm{BBB}$ they affect directly or indirectly composition and function of the BBB, as will be discussed in the next paragraphs (Banks 2005, Banks et al. 1995). Impaired BBB permeability accompanied by altered cytokine composition can be one of the factors leading to normal pressure hydrocephalus (Sosvorova et al. 2015).

\section{Effects of hormones on the composition and function of the BBB: paracellular way}

The $\mathrm{BBB}$ is rich in hormonal receptors of all kinds, both on its membrane and inside the cells. Therefore hormones may affect the properties and function of the barrier by non-genomic as well as genomic mechanism. Generally, hormones and other biologically active substances may act on the BBB in two ways: 1. to influence tight junction integrity and thus change the BBB permeability (paracellular way) and,
2. to affect properties or the expression of their transporters (transcellular way).

Most reports published today on the effects of hormones on the BBB permeability concerned steroids, especially glucocorticoids (GCs) and, to a less extent, estrogens.

The effects of GC on the BBB are an example of their complex antiinflammatory actions. It has been repeatedly demonstrated that GCs increase the tightness of TJ by upregulating expression of TJ proteins such as claudin, occludin and vascular endothelian adherin through their binding to glucocorticoid receptors (GR), rich in the BBB forming endothelial cells (Dietrich 2004, Förster et al. 2008, Salvador et al. 2014). A clear evidence on genomic action of GCs on TJ protein occludin via their receptors in murine capillary endothelial cell was provided by Förster et al. (2005). The authors used immortalized BBB endothelial cells retaining their binding end proteosynthetic as well as bioelectric abilities, enabling to study occludin expression. GCs enhanced it and this effect was completely abolished by GR antagonist mifepristone (RU 486). The experiments have brought another interesting finding that occludin expression by GCs was potentiated by insulin by increasing stabilization of GR protein via insulin signaling cascade.

The positive effect of GCs on tightness of the BBB may be further strengthened by up-regulation of especial tissue inhibitors of the BBB-destroying metalloproteinases. Matrix metalloproteinases (MMPs), out of which the most important is MMP-9 called also gelatinase-B, possess their own tissue inhibitors (TIMMP(s), the expression of which is increased by GCs. Both direct effect as well as via expression of selective cytokines may cause matrix metalloproteases inhibition (Forster et al. 2007). This mechanism resembles another one, responsible for reduction of inflammatory mediators formation (leukotrienes and others) by $\mathrm{GC}$, namely inhibition of phospholipase A2 enzyme, catalyzing hydrolysis of phosphoglycerol esters of arachidonic acid, by up-regulation of its inhibitors, lipocortins (Magrioti and Kokotos 2013).

Glucocorticoids, as mentioned already, exert many of their actions via cytokines, but cytokines themselves influence permeability of the BBB as well through their signaling, especially at neuroinflammation. The BBB is rich of cytokine receptors forming endothelial membrane microdomains. These receptors may- but need not be identical with their transporters. In 
addition, endothelial cells and adjacent glial cell of the BBB are the sites of cytokine biosynthesis. For review of cytokine modulatory effects on the BBB properties and function see e.g. Pan et al. (2011).

Finally, let us mention well known antiinflammatory effect of GCs, taking part also at the $\mathrm{BBB}$, and resulting in reducing of leukocyte infiltration across the disrupted barrier. It consists in counteracting the upregulation of intracellular adhesion molecule-1 and vascular cell adhesion molecule-1 (ICAM-1 and VCAM-1, respectively) (Salvador et al. 2014).

Elucidation of these mechanisms is important for treatment of many neurodegenerative inflammatory diseases such a multiple sclerosis and others by GC (Salvador et al. 2014).

Though not so efficient as glucocorticoids, estrogens are other steroids influencing the permeabiliity of the BBB: estradiol through its receptor in the BBBforming endothelial cell up-regulates expression of occludin in a similar way as GCs (Sandoval and Witt 2011). Either directly via their receptors or indirectly through cytokine signaling estrogens reduce activation of adhesion molecules and consequetly penetration of leukocytes across the barrier (Witt and Sandoval 2014).

Increased expression of tight junction proteins was reported even by natural plant products with mild estrogenic properties as is flavonoid baicalin (Zhu et al. 2012). Since many phytoestrogens and also estrogen-like compounds from the environment, known as endocrine disruptors, may influence the properties and function of the BBB, new studies addressing this topics may be expected.

\section{Effects of hormones on the composition and function of the BBB: transcellular way}

Unlike paracellular effects, so far there are not many reports on the effect of hormones on transport of various substances across the BBB. Most of them were performed with experimental animals or in cell cultures.

Of interest may be the effect of estradiol on the expression of glucose transporter-1 by estradiol in rats (Shi and Simpkins 1997). Both types of estradiol receptors $(\alpha$ and $\beta)$ are present in the endothelial cells of the BBB and through them estradiol regulates expression of several proteins of the ATP-binding cassette (ABC). Among them the most important is breast cancer resistance protein (BCRP). This protein is responsible for extrusion of a variety of therapeutic drugs, including cytostatics, and thus diminishes their pharmacological efficacy in the brain. Estradiol induces a down-regulation of BCRP on transcriptional and translational levels via the activation of ER $\beta$ in rat brain capillaries (Mahringer and Fricker 2010). The function of transporters of the ATP-binding cassette may be also modulated indirectly by cytokines (see above) (Pan et al. 2011).

Glucocorticoids, besides their plethora effects on the BBB permeability are also involved in regulation of key transporters of various substrates and drugs. Out of them already mentioned permeability phosphoglycoprotein or $\mathrm{pgP}$, the product of multidrug resistance gene, is of particular importance, with respect to its neuroprotective role as it actively pumps substrates back into the capillary lumen. Experiments with developing murine fetuses indicated that pgP expression is upregulated by synthetic GCs (Petropoulos et al. 2010).

Expression of polyfunctional transporters operating in (on) the BBB could be also regulated by androgens: Organic anion transporter 3 (OAT3) mRNA in the cultured rat BBB cells was induced by treatment with dihydrotestosterone (DHT) by genomic way through androgen receptors since the effect was completely abolished by antiandrogen flutamide. This protein among others mediates transport of thyroid hormones and steroid conjugates (Ohtsuki et al. 2005).

\section{Conclusion}

There are many reports on effects of hormones in the brain, as well as on their transport across the BBB. Surprisingly, only scarce information is available how they affect BBB composition and their own transport. The endothelial cells composing the BBB are rich in hormonal receptors, both membrane and intracellular.

In this review we tried to outline the main ways how hormones regulate expression of tight junctions and adhesion proteins and thus the BBB permeability, as well as their effects on expression and function of various transporters including those transporting hormones themselves. It concerns first of all glucocorticoids, used for many years as powerful antiinflammatory agents. In general they increase the tightness of the BBB. Their pluripotent effect consists in up-regulation of TJ proteins expression of and, at the same time in down-regulation of destructive enzymes by increasing expression of their specific inhibitors. In addition GCs regulate expression of important protein transporters responsible for removal of detrimental substances from the brain. Of interest are also 
beneficial effects of estrogens and even also androgens, acting in similar way as GCs.

The effects of hormones on the BBB is an open lively topics and new reports addressing it may be expected.

\section{Conflict of Interest}

There is no conflict of interest.

\section{Acknowledgements}

The study was supported by the grant NT/13890-4 from the Internal Grant Agency of the Czech Ministry of Health.

\section{References}

ABBOTT NJ, PATABENDIGE AA, DOLMAN DE, YUSOF SR, BEGLEY DJ: Structure and function of the bloodbrain barrier. Neurobiol Dis 37: 13-25, 2010.

BANKS WA: Blood-brain barrier transport of cytokines: a mechanism for neuropathology. Curr Pharm Des 11: 973-984, 2005.

BANKS WA: Delivery of peptides to the brain: emphasis on therapeutic development. Biopolymers 90: 589-594, 2008a.

BANKS WA: The blood-brain barrier as a cause of obesity. Curr Pharm Des 14: 1606-1614, 2008b.

BANKS WA, KASTIN AJ, BROADWELL RD: Passage of cytokines across the blood-brain barrier. Neuroimmunomodulation 2: 241-248, 1995.

BANKS WA, OWEN JB, ERICKSON MA: Insulin in the brain: there and back again. Pharmacol Ther 136: 82-93, 2012.

BARROS LF, BITTNER CX, LOAIZA A, PORRAS OH: A quantitative overview of glucose dynamics in the gliovascular unit. Glia 55: 1222-1237, 2007.

BJÖRKHEM I: Crossing the barrier: oxysterols as cholesterol transporters and metabolic modulators in the brain. J Intern Med 260: 493-508, 2006.

DIETRICH JB: Endothelial cells of the blood-brain barrier: a target for glucocorticoids and estrogens? Front Biosci 9: 684-693, 2004.

FÖRSTER C, SILDWEDEL C, GOLENHOFEN N, BUREK M, KIETZ S, MANKERTZ J, DRENCKHAHN D: Occludin as a direct target for glucocorticoid-induced improvement of bloo-brain barrier properties in a murine in vitro system. J Physiol 565: 475-486, 2005.

FÖRSTER C, KAHLES T, KIETZ S, DRENCKHAHN D: Dexamethasone induces the expression of metalloproteinase inhibitor TIMP-1 in the murine cerebral vascular endothelial cell line cEND. J Physiol 580: 937-949, 2007.

FÖRSTER C, BUREK M, ROMERO IA, WEKSLER B, COURAUD PO, DRENCKHAHN D: Differential effects of hydrocortisone and TNFalpha on tight junction proteins in an in vitro model of the human blood-brain barrier. J Physiol 586: 1937-1949, 2008.

HAGENBUCH B, MEIER PJ: The superfamily of organic anion transporting polypeptides. Biochim Biophys Acta 1609: 1-18, 2003.

HAMPL R, BIČÍKOVÁ M, SOSVOROVÁ L: Hormones and the blood-brain barrier. Horm Mol Biol Clin Investig 21: 159-164, 2015.

HAWKINS RA, O'KANE RL, SIMPSON IA, VIÑA JR: Structure of the blood-brain barrier and its role in the transport of amino acids. $J$ Nutr 136 (Suppl 1): 218S-226S, 2006.

JEITNER TM, VOLOSHYNA I, REISS AB: Oxysterol derivatives of cholesterol in neurodegenerative disorders. Curr Med Chem 18: 1515-1525, 2011.

KALRA SP, KALRA PS: Neuroendocrine control of energy homeostasis: update on new insights. Prog Brain Res 181: $17-33,2010$.

LÖSCHER W, POTSCHKA H: Blood-brain barrier active efflux transporters: ATP-binding cassette gene family. NeuroRx 2: 86-98, 2005.

MAGRIOTI V, KOKOTOS G: Phospholipase A2 inhibitors for the treatment of inflammatory diseases: a patent review (2010-present). Expert Opin Ther Pat 23: 333-344, 2013. 
MAHRINGER A, FRICKER G: BCRP at the blood-brain barrier: genomic regulation by 17ß-estradiol. Mol Pharm 7: 1835-1847, 2010.

OHTSUKI S, TOMI M, HATA T, NAGAI Y, HORI S, MORI S, HOSOYA K, TERASAKI T: Dominant expression of androgen receptors and their functional regulation of organic anion transporter 3 in rat brain capillary endothelial cells; comparison of gene expression between the blood-brain and -retinal barriers. J Cell Physiol 204: 896-900, 2005.

PAN W, KASTIN AJ: Adipokines and the blood-brain barrier. Peptides 28: 1317-1330, 2007.

PAN W, STONE KP, HSUCHOU H, MANDA VK, ZHANG Y, KASTIN AJ: Cytokine signaling modulates bloodbrain barrier function. Curr Pharm Des 17: 3729-3740, 2011.

PETROPOULOS S, GIBB W, MATTHEWS SG: Developmental expression of multidrug resistance phosphoglycoprotein (P-gp) in the mouse fetal brain and glucocorticoid regulation. Brain Res 1357: 9-18, 2010.

RAMALINGAM M, KIM SJ: Mechanisms of action of brain insulin against neurodegenerative diseases. $J$ Neural Transm 121: 611-626, 2014.

SALVADOR E, SHITYAKOV S, FÖRSTER C: Glucocorticoids and endothelial cell barrier function. Cell Tissue Res 355: 597-605, 2014.

SANDOVAL KE, WITT KA: Age and 17 $\beta$-estradiol effects on blood-brain barrier tight junction and estrogen receptor proteins in ovariectomized rats. Microvasc Res 81: 198-205, 2011.

SHEN S, ZHANG W: ABC transporters and drug efflux at the blood-brain barrier. Rev Neurosci 21: 29-53, 2010.

SHI J, SIMPKINS JW: 17 beta-Estradiol modulation of glucose transporter 1 expression in blood-brain barrier. Am J Physiol 272: E1016-E1022, 1997.

SMITH QR: Transport of glutamate and other amino acids at the blood-brain barrier. J Nutr 130 (4S Suppl): 1016S1022S, 2000.

SOSVOROVA L, MOHAPL M, VCELAK J, HILL M, VITKU J, HAMPL R: The impact of selected cytokines in the follow-up of normal pressure hydrocephalus. Physiol Res 64 (Suppl 2): S283-S290, 2015.

TAJES M, RAMOS-FERNÁNDEZ E, WENG-JIANG X, BOSCH-MORATÓ M, GUIVERNAU B, ERASO-PICHOT A, SALVADOR B, FERNÀNDEZ-BUSQUETS X, ROQUER J, MUÑOZ FJ: The blood-brain barrier: Structure, function and therapeutic approaches to cross it. Mol Membr Biol 31: 152-167, 2014.

WANG H, ECKEL RH: What are lipoproteins doing in the brain? Trends Endocrinol Metab 25: 8-14, 2014.

WIRTH EK, SCHWEIZER U, KÖHRLE J: Transport of thyroid hormone in brain. Front Endocrinol (Lausanne) 5: 98, 2014.

WITT KA, SANDOVAL KE: Steroids and the blood-brain barrier: therapeutic implications. Adv Pharmacol 71: 361-390, 2014.

ZHU H, WANG Z, XING Y, GAO Y, MA T, LOU L, LOU J, GAO Y, WANG S, WANG Y: Baicalin reduces the permeability of the blood-brain barrier during hypoxia in vitro by increasing the expression of tight junction proteins in brain microvascular endothelial cells. J Ethnopharmacol 141: 714-720, 2012. 